\title{
VIRTUAL BLOXING - ASSEMBLY RAPID PROTOTYPING OF NEAR NET SHAPES
}

\author{
Theodore Lim \\ Heriot-Watt University, Edinburgh, Scotland, UK \\ Tel: $+44(0) 1314495111$ \\ t.lim@hw.ac.uk
}

\author{
Hugo Medellín \\ Facultad de Ingeniería, Universidad Autónoma de \\ San Luis Potosí, S.L.P., México \\ Tel: +5244482623 30 \\ hugoivanmc@uaslp.mx
}

\author{
Raymond Sung \\ Heriot-Watt University, Edinburgh, \\ Scotland, UK \\ Tel: $+44(0) 1314495111$ \\ r.c.w.sung@hw.ac.uk
}

\author{
James Ritchie \\ Heriot-Watt University, Edinburgh, \\ Scotland, UK \\ Tel: +44 (0)131 4495111 \\ J.M.Ritchie@hw.ac.uk
}

\author{
Jonathan Corney \\ University of Strathclyde, Glasgow, \\ Scotland, UK \\ Tel: +44 (0)141548 2254 \\ jonathan.corney@strath.ac.uk
}

\begin{abstract}
Virtual reality (VR) provides another dimension to many engineering applications. Its immersive and interactive nature allows an intuitive approach to study both cognitive activities and performance evaluation. Market competitiveness means having products meet form, fit and function quickly. Rapid Prototyping and Manufacturing (RP\&M) technologies are increasingly being applied to produce functional prototypes and the direct manufacturing of small components. Despite its flexibility, these systems have common drawbacks such as slow build rates, a limited number of build axes (typically one) and the need for post processing. This paper presents a Virtual Assembly Rapid Prototyping (VARP) project which involves evaluating cognitive activities in assembly tasks based on the adoption of immersive virtual reality along with a novel nonlayered rapid prototyping for near net shape (NNS) manufacturing of components. It is envisaged that this integrated project will facilitate a better understanding of design for manufacture and assembly by utilising equivalent scale digital and physical prototyping in one rapid prototyping system. The state of the art of the VARP project is also presented in this paper.
\end{abstract}

Keywords: Virtual Reality, Rapid prototyping, Assembly planning, Near net shape.

\section{INTRODUCTION}

The success of a manufacturing business enterprise not only requires short product inception but also necessitates the integration of engineering, marketing and production components. Therefore it is important that manufacturing planning and analysis methods work within the framework of the product design cycle [1]. To improve the value added to a product, technology development is critical and tools such as virtual reality (VR) and rapid prototyping (RP) can provide combined advantages to manufacturing enterprises.

Rapid prototyping is a manufacturing process where preproduction models are built to test various aspects of a design's functionality. RP is an additive process in which parts are built by adding material layer upon layer as opposed to traditional techniques such as machining which is subtractive (i.e. removing material). Wholers [2] indicated that $27.3 \%$ of the RP parts are used as functional models and fit and assembly, meanwhile, visual applications account for $26.3 \%$. More than $29 \%$ of customers use the parts for tooling components, such as mold inserts, and patterns for urethane and metal castings. Meanwhile, rapid manufacturing-the direct production of finished goods from additive fabrication-account for $9.6 \%$.

The creation of assemblies is also fundamental to modern manufacturing economies. Indeed, around $40 \%$ of a product's cost has been attributed to the assembly stage [3]. Assembly planning traditionally has been an intuitive, heuristic and lengthy process based upon the skills and subjective experience of an 'expert' planner. This expert produces a formal assembly plan usually after most of the important design, and manufacturing decisions are finalised.

This paper presents how VR can be used as a tool to evaluate and improve the efficiency of the RP process. It briefly outlines the current state-of-the-art in assembly planning research and describes an investigation into the use of Virtual Environments (VEs) to address this problem. This has led to the proposal of a novel approach utilising immersive Virtual Reality to enhance the assembly expert's role during the planning process. A novel non-layered RP\&M technique based on direct interaction with $3 \mathrm{D}$ solid models, $\mathrm{CNC}$ and robotic assembly is 
then outlined; the system under development, referred to as RPBloX, has the potential to either supplement current RP techniques or act as a standalone method. Although both feasibility studies are being investigated separately, they are integrated to form the Virtual Assembly Rapid Prototyping (VARP) test bed.

\section{RESEARCH RELATED WORK}

Traditionally assembly plans were generated only after the product design had been completed, approved and authorized. Assembly guidelines typically comprise a combination of experiential, analytical and theory-base recommendations. Human factors are decisive in assembly rationalization. In the last years, assembly planning has been subject to significant research efforts with most work concentrating on attempting to semi- or automate the sequencing process. Bekey [4] reports that these efforts have had limited success outside the research community. This is due to a number of reasons:

- Algorithms are computationally expensive for realistic assemblies.

- Narrow selection criteria means multitude of sequences generated by such systems detract from the credibility of the results.

- There is a natural delay in the transfer of knowledge to industry.

Common research relating to assembly planning automation includes sequence representation, generation, evaluation and optimisation; process accuracy and efficiency, and motion planner integration [5]. Assembly sequence generation has primarily focused on algorithms for the fast and efficient generation of feasible assembly plans. Most of the assembly sequence generators transform the problem of generating assembly sequences into the problem of generating disassembly sequences. To evaluate the feasibility of an assembly sequence, various issues must be considered: geometrical, mechanical, manipulability, accessibility, stability, visibility, and material. The geometrical feasibility is one of the most important criteria to evaluate an assembly sequence because it validates collision between parts [6].

Bourjault [7] made a significant early contribution to assembly planning by proposing an interactive method based on liaison precedence to generate all valid assembly sequences for a product. Homem and Sanderson [8] used AND/OR graphs to show all the possible assembly operations including subassembly configurations, thus substantially reducing the size of the data tree required to represent the full set of assembly sequences. Disassembly analysis is also claimed as an attribute of this approach. Latombe et al. [9] described assembly sequencing as "intractable" and claim that this has caused contemporary researchers to restrict their work to small areas of interest in the field limited to small assembly families with tightly controlled product representations.

In terms of optimising sequences Baldwin et al. [10] reduce the number of sequences by displaying different components in their assembled state on a computer screen; users then choose and delete the undesirable ones. This work is taken a step further by Wilson [11] who uses a CAD model to answer most of the precedence type questions then the user eliminates interactively, undesirable states. He contends that, for the foreseeable future, human intuition is necessary to make decisions on some of the assembly constraints. It can be noted in passing that the systems described based on interrogation of CAD models are generally concerned with assemblies comprising rigid components. This is a significant limitation in the long run when it is remembered that many assemblies include flexible and deformable components; a prime example in electro-mechanical products is the cable harness.

Automation of process planning in RP is also an emerging research topic. In RP a significant amount of human intervention is required to produce an optimal part, a feature of the process similar to assembly planning. Process planning is therefore required to determine build orientation, support and part specific fixturing or tooling, to generate slicing and selection process parameters and to minimise human intervention [12].

The use of VR tools has been recently introduced to provide three-dimensional input and stereoscopic viewing to perform, verify, and plan the assembly of components, and for training purposes. Virtual reality environment offers a more natural, powerful, economic, flexible platform than a traditional engineering environment to support assembly planning [13]. Several investigations into virtual environments for design and assembly tasks make extensive use of advanced computer graphics. However, screen-based CAD/CAM, which only stimulates the visual senses, has made actual physical contact during product development an increasingly rare occurrence. Engineers still find the sensation of handling a physical prototype or experiencing manufacturing processes useful and intuitive; indeed, hands-on experience reinforces the understanding of the physical, operational and visual aspects of engineered items [14]. To facilitate this, the sense of touch and kinesthesia have been introduced in computer based design and manufacturing environments by using haptic devices, (for instance see [15]-[16]), which enables manual force-feedback interaction with virtual environments or teleoperated remote systems. Force feedback devices, such as SensAble's Phantom Haptic Device [17] has been a research topic in the development of advanced computer-aided design systems, gaming and other simulated environments. However, very little work has been done towards the development of haptic humancomputer interface for assembly process planning. 


\section{VIRTUAL REALITY IN RAPID PROTOTYPING AND ASSEMBLY}

Immersive VR is an attractive option since it offers the user a sense of being immersed in the data where objects have a sense of 'presence' [18] and allows them to interface with the data at full scale if required. A design, after all, begins with an image or idea and the concept is disseminated via hand gestures, diagrams and descriptive speech. Depending on the industry, it is almost certain that pre-production prototypes are required, either as virtual or physical artefacts. These prototypes can be near net shapes. Cost is always a factor and whilst a physical prototype may ultimately be needed, its virtual counterpart is studied to improve product design, quality and performance, assembly and other issues. This reduces manufacturing risks of prototypes early in the product development cycle, and consequently reduces the number of costly design-build-test cycles [19].

The quality requirements in RP demand a significant degree of expertise, especially where consistency is concerned. Design by virtue is an iterative process and building prototypes can be costly, often based upon trial-and-error. Although computer based manufacturing systems provide useful tools to prove conceptual designs its interaction is usually limited to a $2.5 \mathrm{D}$ plane.

So can VR be beneficial to RP process and who needs it? VR has been suggested as a complementary technology to RP [20]. It is expected that the attributes of VR and RP will lead to more efficient product development. Another contribution of VR in RP is the visualisation of support structures and for correcting STL List files [21,22]. Surveys and review articles have indicated that virtual prototyping is both attractive and beneficial, [23,24]. For novice RP-users, virtual environment systems would help them gain a better global view of RP technology. The virtual environment can also assist take in of well-founded decisions for investment or even outsourcing of RP tasks to meet the quick and diverse appearance of new and improved processes. However, it is the contention of this paper that VR has an important role to play as a front end to the development of physical prototypes, especially in the assemblyplanning domain.

An important observation from the plethora of literature on process planning systems is the lack of practical use in industry. Although academically challenging and effective for tightly restricted or very simple families of assemblies, the nature of the work is such that industrial testing is seldom attempted. Burgoyne and Murray [25] describe some early solutions to the problems of applying modelling in industrial situations and reusing $\mathrm{CAD}$ and other design information. This is typical of how many companies have since applied multi-media and other modern 3D CAD tools to their assembly planning solutions. The systems developed certainly make good use of the interactive nature of computer software but do not incorporate assembly intelligence, assembly intent or any means by which assembly sequences can be automatically generated during or after assembly planner use.

Work by Ritchie et al [26], has shown that immersive VR can be used to effectively generate assembly sequences and process plans. It was also apparent that, in comparison to CAD environments, immersive VR offered an unrestrained, real-time means of interaction at full scale; other scales being possible if necessary. The system utilised 3D CAD models and user interaction as its key inputs. The combination of the latter including the recording activities carried out during the virtual assembly process forms the basis for VARP.

\section{VIRTUAL ASSEMBLY RAPID PROTOTYPING}

VARP is essentially being developed on two fronts, i.e. rapid prototyping and virtual assembly. The significance of VARP is in the utilisation of VR to support the design and rapid prototyping of large scale prototypes. The platform also addresses issues relating to design for manufacture and assembly (DFMA) while human factors are considered by evaluating cognitive activities as the user interacts within the virtual environment.

\section{RAPID PROTOTYPING WITH RPBLOX}

RPBloX follows the use of conventional workshop machinery and technology [27]. The RPBloX methodology involves a cellular approach to building up a part. Rather than slicing up the CAD model into numerous thin sheets, RPBloX segments the model into 3D cells (or Blox) of varying sizes. On the main advantages of the RPBloX system over other RP techniques is the reduction of the build time especially when large and/or tick wall components are fabricated. Consequently, production costs could be significantly reduced without the forfeiting accuracy and timeliness.

\subsection{RPBloX architecture}

The architecture has three main steps: discretization of 3D models into Bloxs; perform DFMA; generate machining and robotic assembly code for manufacturing. The user interface allows interactive decomposition of a digital model, assembly sequencing and modifications to Blox units. The architecture is open and additional functions to support manufacturability and assembly assessment can be easily added. For example, a fitness test can be applied to the Blox set to determine which Blox within the set would pose manufacturing difficulties or perhaps prevent robot pick up. Figure 1 presents an overview of the RPBloX system. 


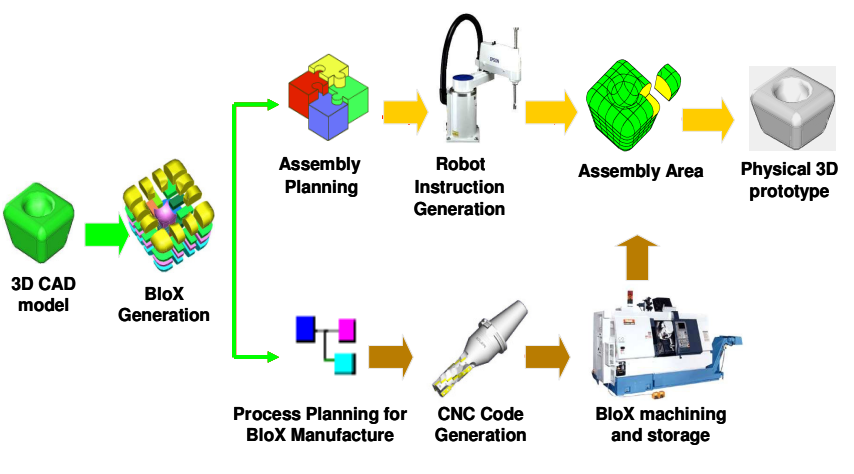

Figure 1. Overview of the RPBloX system.

\subsection{Subdivision methods and Blox generation}

Unlike 2D slicing of an STL model, RPBloX works directly with a $3 \mathrm{D}$ model and allows mixed slicing in any orientation. For full interactive slicing users can select from axis-aligned means to entity (e.g. face, vertex) selection methods to generate any number of slicing planes as required (Figure 2).

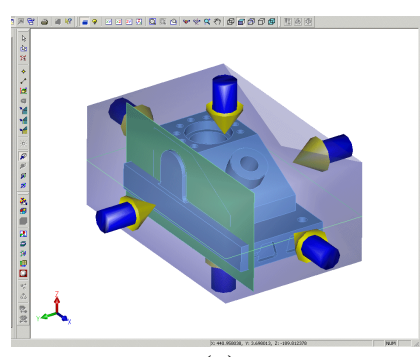

(a)

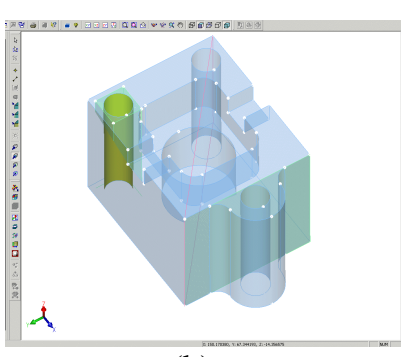

(b)
Figure 2. Interactive subdivision. (a) Axis-aligned in conjunction with view-cube. (b) Entity selection method.

Various automatic and semi-automatic slicing algorithms have also been incorporated. Loops and isolines as segmentation tools for prismatic and complex curved surface components are extremely powerful. The Iso-Loop decomposition algorithm identifies holes and bosses on a face. When a boss is found, a slicing plane with the same surface type as the face on which the loop lies, is used to separate the boss at its base.

The use of isolines is twofold; the main use is in the segmentation of curved faces. Since isolines lie on the surface they can also be used to detect inflexion curvatures on complex surfaces. Using the isoline as a guide a slicing plane is generated and the component segmented. Figure 3 shows the results of decomposition using the iso-loop method. The example component has several similarities to medical implants such as hip replacements units.
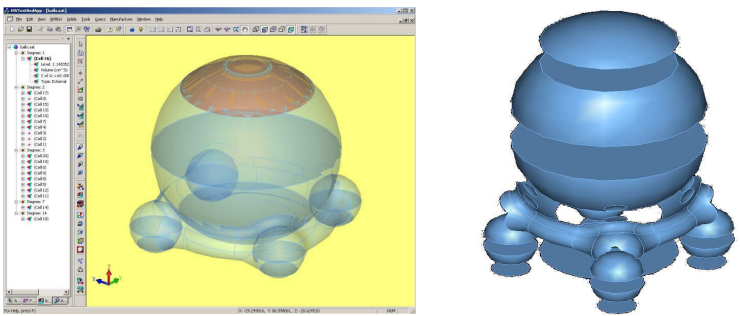

Figure 3. Decomposition using the Iso-Loop decomposition.

A pattern can then be applied to the component for further decomposition. Patterns are repeating shapes and in this case the profile of a cross-shaped bar stock is used (Figure 4a). The end result is a Blox set and the prototype that can be assembled like stacking bricks.

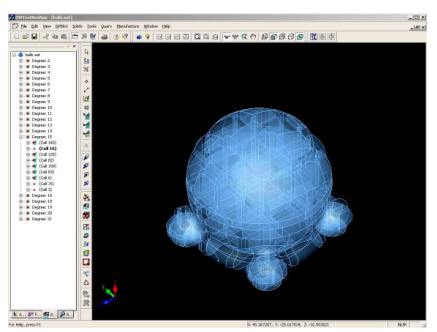

(a)

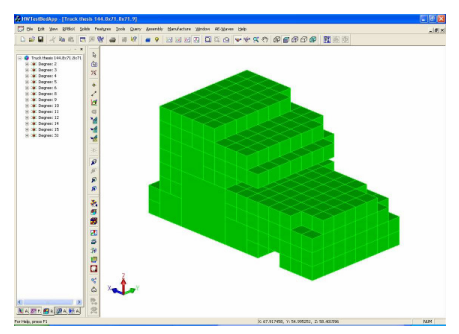

(b)
Figure 4. Decomposition: (a) Pattern, (b) Octree.

RPBloX also supports Octree decomposition which is a hierarchical form of tessellation. A number of well known Octree algorithms for subdivision, neighbour finding and tree traversal have been implemented (Figure 4b) [28]. Combined methods for the generation of Blox are also available in the RPBloX system. Figure 5 presents a decomposition of a 3D model using a combination of iso-loop and pattern methods.

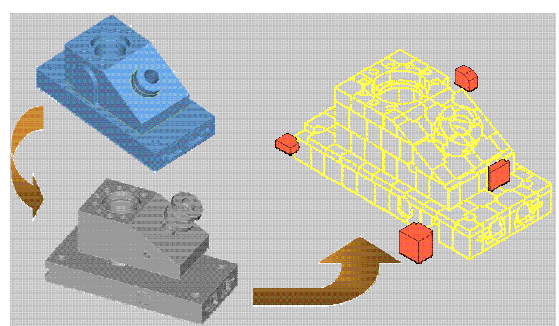

Figure 5. Iso-loop \& pattern decomposition.

\subsection{Machining and robotic assembly code}

The generation of CNC code for machining the Blox set is an ongoing work. Some of the results have shown that CNC code for machining the Blox set can be generated (Figure 6). 


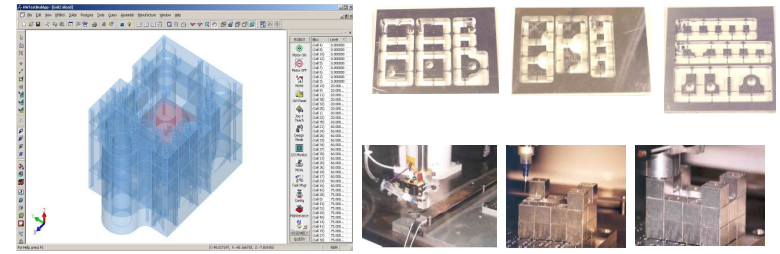

Figure 6. Machining of Blox using the RPBloX data.

Assembly planning and automatic generation of robotic instructions have been implemented in the RPBloX system. As a result, an integrated subsystem for the production of near net shape components based on the Octree decomposition of 3D models has been developed (Figure 7a). The subsystem RPNNS (rapid production of near net shapes) [29], works by subdividing a 3D model into cubes of various sizes using an Octree decomposition and optimisation method to reduce the number of cubes required without affecting the accuracy of the approximation. Once the Octree model has been verified for physical fabrication, the system generates instructions for a robot to construct the NNS model (Figure 7b).

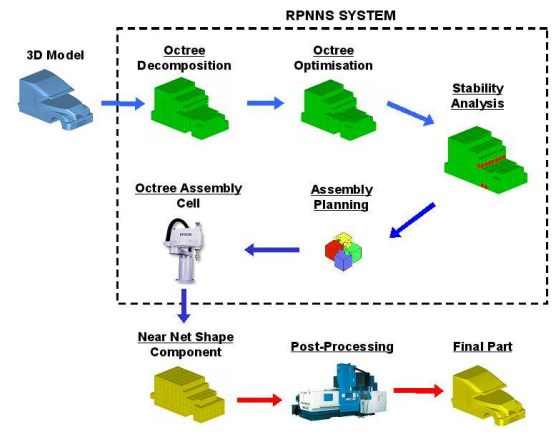

(a)

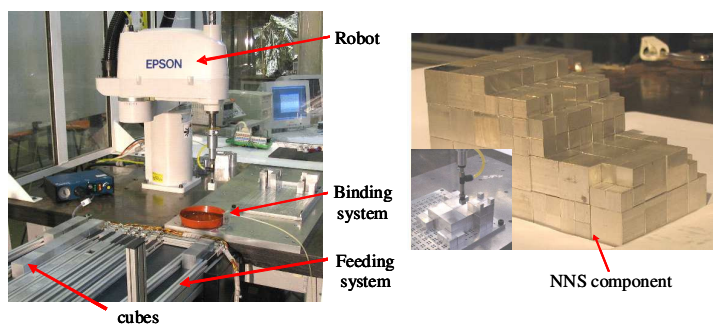

(b)

Figure 7. (a) The RPNNS system (b) NNS prototyping.

\section{VIRTUAL ASSEMBLY PLANNING}

Two experimental platforms have been developed to investigate assembly planning in virtual reality. These two platforms, named as COSTAR (Cable Organisation System Through Alternative Reality) and HAMMS (Haptic Assembly, Manufacturing and Machining System), are presented in this section.

\subsection{COSTAR}

The COSTAR project began in 2001 to investigate the benefits of using VR to design and route cable harnesses [30]. The results showed that VR gave productivity benefits over CAD during creative cable routing design activities. Follow-on work was aimed at understanding how various aspects of the immersive VR system were contributing to these benefits and how engineering design and planning processes could be analysed as they are being carried out. This was performed using comprehensive user-logging to non-intrusively collect detailed information relating to design solutions and assembly planning approaches used by a number of engineers. The COSTAR system logs all the user's interactions in the virtual environment. The contents of the log file includes the user's head and hand positions, menu options selected, design changes made together with a timestamp for each logged event. User tests have been performed to evaluate the effectiveness of the system and also to compare its performance with traditional 2D CAD systems, [31,32].

In COSTAR the user wears a Virtual Research V8 headmounted display (HMD) and Pinch ${ }^{\circledR}$ Gloves to operate the system, Figure 8. To track the user's head and hand movements, an Ascension Flock of Birds ${ }^{\circledR}$ electromagnetic tracking system is used. The VR application was developed using SENSE8®'s WorldToolKit ${ }^{\circledR}$ release 9.

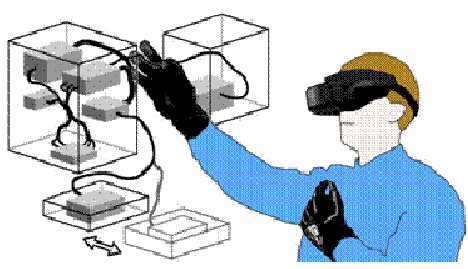

Figure 8. The COSTAR planning system.

When the user carries out a design task in the virtual environment, all their actions are logged into a text file. Since each logged event has a timestamp, chronological account of what happened during the session and how long the user spent on each specific task can be done. Automatic post-processing of the logged text file has been automated so a large amount of files can be processed to extract assembly plans.

\subsection{HAMMS}

The HAMMS (Haptic Assembly, Manufacturing and Machining System) system has been developed as a test bed to investigate the user interactions and response while performing various engineering tasks using a haptic device in a virtual environment. The hardware comprises a Phantom haptic device and uses a pair of CrystalEyes ${ }^{\circledR}$ stereoscopic glasses for stereo viewing. The systems' architecture is presented in Figure 9. 


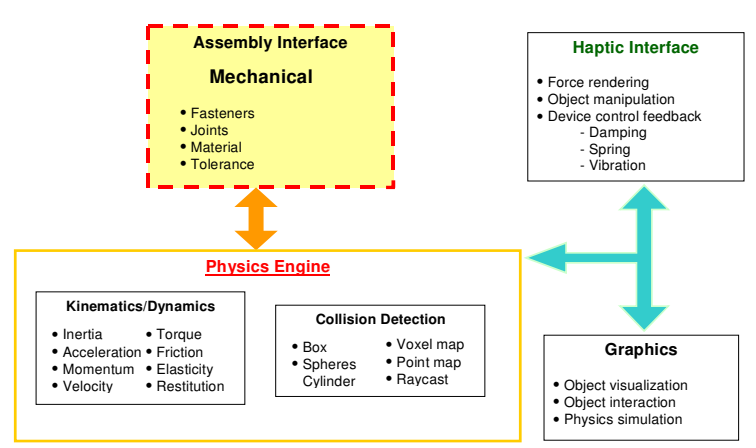

Figure 9. HAMMS architecture.

The HAMMS environment allows objects to have their physical state modified and user/device/object interactions logged in real-time. The basic logged data comprises position, orientation, time stamps, velocity and an object index. Figure 10 illustrates the HAMMS GUI and the color-coded therblig units adapted by HAMMS. To visualize the data stream, large spheres are used to signify the start of an event, while smaller contiguous spheres indicate the direction, speed, and location of exploration or controlled displacements. Green indicates search, find or rest. Blue represents selection and inspection. Red identifies control events such as grasping, holding, translation, dis/assembly operations. Velocity changes are indicated by the separation of the spheres, i.e. sparsely spaced spheres equate to higher velocity. The line joining all spheres is referred as the motion-time-line (MTL). Detailed information regarding HAMMS can be found in [33].

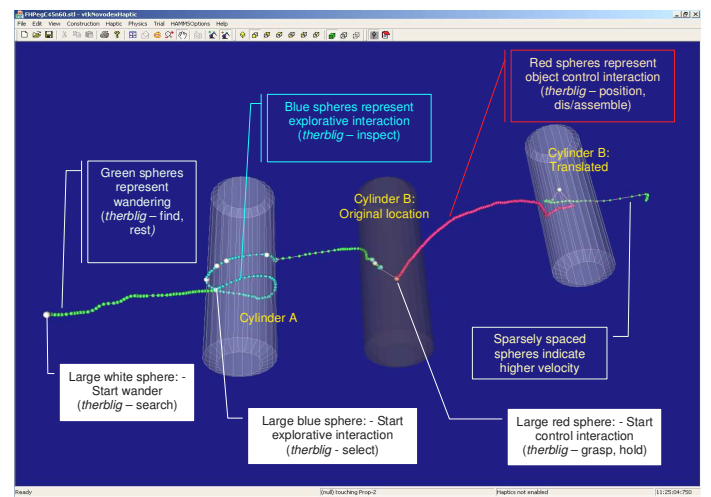

Figure 10. HAMMS user interface

The logged data in the HAMMS system is stored as a text file which is then used to extract and generate the assembly plan. This file contains information of the therblig units associated with the virtual assembly process. By using a data parser, assembly operations can be identified, filtered and extracted to generate assembly plans as shown in Figure 11.

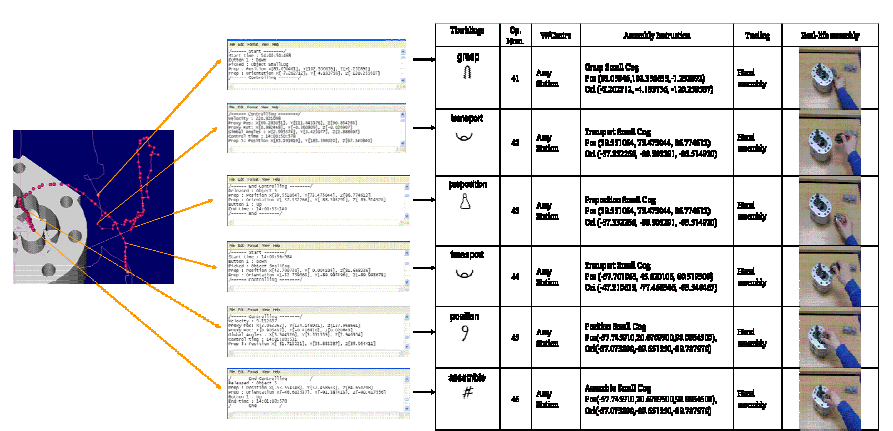

Figure 11. Identification and extraction of assembly operations.

The results of the tests carried out to evaluate the HAMM systems have clearly shown that the chronocycle data and the logged file can be used to identify individual assembly tasks and motions performed by the user during the assembly. These assembly details can be further used to automatically generate assembly plans associated to physical operations.

\section{THE VARP SYSTEM}

The classical context regarding assembly sequences has been to try to replace the human planner with systems which attempt to take most or all of the decision making away from the expert and replace their function with semi-automated or automated systems. In contrast VARP allows the user to interactively decompose models, perform design changes, analyse assembly processes, analyse manufacturing processes, carry out assembly tasks, evaluate cognitive design and manufacturing activities, and produce assembly and manufacturing plans via an immersive virtual and haptic interface. An overview of the proposed VARP system is presented in Figure 12.

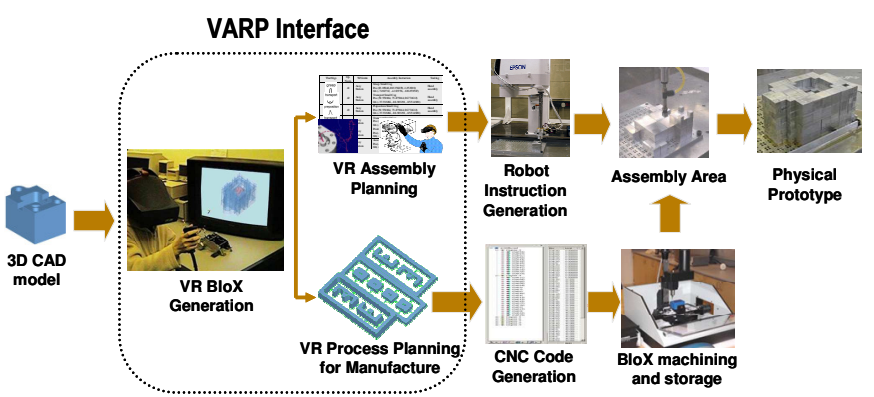

Figure 12. The Virtual Assembly Rapid Prototyping system.

The advantage of VARP is its unique sensory environment to explore and analyse assembly options. It will also enable walk-through so that one can see the build process, the pit falls of a portion of the design, and to make amendments before production. By collecting and analysing data in real-time, process parameters can be validated and subsequently applied or transferred to real manufacturing machines such as $\mathrm{CNC}$ 
machines, robots or RP machines. From this rich data source, cognitive evaluation of design and manufacturing processes can be used to push information to novice users and burgeoning designers.

\section{CONCLUSIONS}

This paper presents an alternative approach based on the adoption of immersive virtual reality and haptic interface for design and manufacture that can be used to produce practical downstream manufacturing information for non-layered physical prototypes. The development of a virtual reality-based assembly system can shorten the product innovation cycle and capitalise the experience of assembly operators. By analysing expert usage within the system a better understanding of the implications of virtual technologies and the cognitive association to product design can be gathered.

The elements of VARP are a short step away from full integration. RPBloX and the virtual systems COSTAR and HAMMS will form the basis of the unified platform. Further developments are also earmarked in the area of Blox manufacturing planning, analysis for rapid prototyping and manufacturability and on geometric evaluations.

Finally, the unique combination of digital and physical prototyping will enable various forms of prototyping to be evaluated within the context of the product development.

\section{ACKNOWLEDGMENTS}

We would like to acknowledge funding of this work by the UK EPSRC IMRC grant GR/R35285/01. The authors also gratefully acknowledge the support of numerous industrial partners involved in the project and the Fund for Research Support (FAI) of the UASLP and the Secretaria de Educacion Publica of México.

\section{REFERENCES}

1. McLeod, J. Superfast simulators make it a lot easier to skip prototyping. Electronics, 1988, 60(11), 61-68.

2. Wholers, T., Rapid prototyping and manufacturing: State of the industry - Annual World Wide Progress Report, 2006, Wohlers Associates.

3. Bullinger, H.J. and Richter, M. Integrated Design and Assembly Planning. Computer Integrated Manufacturing Systems, 1991, 4(4), 239-247.

4. Bekey, G.A. Foreword (from Computer-Aided Mechanical Assembly Planning, Ed. Homem de Mello L.S., Lee S.), 1991 (Kluwer Academic Publishers, Norwell, Massachusetts), ix-xi.
5. Homem de Mello Luiz S., Lee Sukhan, "Computer-Aided Mechanical Assembly Planning", foreword by George A. Bekey, Kluwer Academic Publishers, 1991.

6. H. Medellín, J. Corney, J.M. Ritchie, T. Lim, J.B.C. Davies, A Novel Approach to the Generation of Automatic and Manual Assembly Plans Using Octrees, IEEE Proceedings of the Fourth Electronics, Robotics and Automative Mechanics Conference (CERMA 2007), September 25-28, 2007, pp.614-619.

7. Bourjault, A. Contribution a une approche méthodologique de l'assemblage automisé:Elaboration automatique des séquences opératoires. PhD Thesis, 1984 (l'Université de Franche-Compté, France).

8. Homem de Mello, L.S. and Sanderson, A.C. AND/OR Graph Representation of Assembly Plans. IEEE Transactions on Robotics and Automation, 1990, 6(2), 188-199.

9. Latombe, J-C, Wilson, R.H. and Cazals, F. Assembly Sequencing with Toleranced Parts. Computer Aided Design, 1997, 29(2), 159-174.

10. Baldwin, D.F., Abell, T.E., Lui, M.M., De Fazio, T.L. and Whitney, D.E. An Integrated Computer Aid for Generating and Evaluating Assembly Sequences for Mechanical Products. IEEE Transactions on Robotics and Automation, 1991, 7(1), 78-94.

11. Wilson, R.H. Minimizing User Queries in Interactive Assembly Planning. IEEE Transactions on Robotics and Automation, 1995, 11(2), 308-312.

12. Bourell, D.L., Beaman, J.J., Marcus, H.L., and Barlow, J.W. Solid freeform fabricationL an advanced manufacturing approach. Proceedings of the SFF Symposium, 1990, pp. 1-7.

13. Ye Nong, Banerjee Prashant, Banerjee Amarnath, and Dech Fred, A Comparative Study of Assembly Planning in Traditional and Virtual Environments. In IEEE Transactions on Systems, Man, and Cybernetics-Part C: Applications and Reviews, Vol. 29, No. 4, 1999.

14. Lim T., Ritchie J.M., Dewar R.G., Corney J.R., Wilkinson P., Calis M., Desmulliez M., Fang J.J., Factors affecting user performance in haptic assembly. In Virtual Reality, Vol. 11, No. 4, 2007, Springer.

15. Howard B.M., Vance J.M., Desktop haptic virtual assembly using physically based modeling. In Virtual Reality, Vol. 11, No. 4, 2007, Springer.

16. Garbaya Samir, Zaldivar-Colado U., The affect of contact force sensations on user performance in virtual assembly tasks. In Virtual Reality, Vol. 11, No. 4, 2007, Springer.

17. Massie T. and Salisbury K., The PHANTOM Haptic Interface: A Device for probing Virtual Objects. In ASME 
Winter Annual Meeting, DSC-Vol. 55-1, pp. 295-300, 1994.

18. Lanzagorta, M., Rosenberg, R., Rosenblum, L.J., and Kuo, E.Y. Rapid prototyping of virtual environments. Computing in Science \& Engineering, 2(3), 2000, pp. 6873.

19. Choi, S.H. and Samavedam, S. Visualisation of rapid prototyping. Rapid Prototyping Journal, 7(2), 2001, pp. 99-114.

20. Gibson, I., Brown, D., Cobb, S., and Eastgate, R. Virtual Reality and Rapid Prototyping. Proceedings of IEE workshop on Virtual Reality in Engineering, 1953, 51-63.

21. Fadel, G.M., Crane, D., Dooley, L., and Geist, R. Support structure visualisation in a Virtual Reality environment. Proceedings of the 6th Int'1 Conf. On Rapid Prototyping, Dayton, 1995.

22. Morvan, S.M. and Fadel, G.M. IVCES: interactively correcting STL files in a virtual environment. Proceedings of the 1996 ASME Design and Engineering Technical Conference, 1996.

23. Zheng, J.M., Chan, K.W., and Gibson, I. Virtual reality A real world review on a somewhat touchy subject. IEEE Potentials, 17(2), 1998, pp. 20-23.

24. Zorriassatine, F., Wykes, C., Parkin, R., and Gindy, N. A survey of virtual prototyping techniques for mechanical product development. Proceeding Instn. Mech. Engrs Part B: J. Engineering Manufacture, 2003, 217, pp. 513-530.

25. Burgoyne, D. and Murray, J.L. The Specification and Development of a Computer Aided Assembly Planning System Suitable for Electro-Mechanical Products. 5th International Conference on Computer Aided Production Engineering, Edinburgh, 1989, pp. 1-4.

26. Ritchie, J.M., Dewar, R.D., Simmons, J.E.L. The Generation and Practical Use of Plans for Manual Assembly using Immersive Virtual Reality Journal of Engineering Manufacture, IMechE Part B, 1999, v213, pp. 461-474.

27. T. Lim, J. Corney, J.M. Ritchie, and B.J. Davies, "RPBloX rapid prototyping - More than just layers", Proc. DETC'02 ASME, Montreal, Canada, 2002, DETC2002/DFM34165.

28. Medellín H., Corney J., Davies J.B.C., Lim T., Ritchie J. M., "Algorithms for the physical rendering and assembly of octree models", Computer Aided Design, 38, 2006, pp. 69-85.

29. H. Medellín, J. R. Corney, J. B. C. Davies, T. Lim, and J. M. Ritchie, Octree-Based Production of Near Net Shape Components, IEEE Transactions on Automation Science and Engineering, vol. 5, no. 3, July 2008, pp. 457-466.
30. Ng FM, Virtual Reality and Computer-Based Tools for the Routing of Cable Harnesses. PhD Thesis 1999, HeriotWatt University, Edinburgh, UK.

31. Ritchie J. M., Sung R. C. W., Robinson G., Day P. N., Dewar R. G. and Simmons J. E. L., Cable harness design, assembly and installation using immersive virtual reality, Virtual Reality Journal, 11, 2007, 261-274.

32. Robinson G., Ritchie J. M., Day P. N. and Dewar R. G.: System design and user evaluation of CoStar: an immersive stereoscopic system for cable harness design, Computer-Aided Design, 2007, 39, 245-257.

33. Ritchie J. M., Lim T., Sung R.S., Corney J.R., Rea H., The Analysis of Design and Manufacturing Tasks Using Haptics and Immerse VR: Some case Studies'. In Talaba Doru and Amditis Angelos (eds.): Product Engineering: Tools and Methods Based on Virtual Reality, 2008, 507522. Springer, Netherlands. 